\title{
A QUASI-STATIC INTERFACE DAMAGE MODEL WITH FRICTIONAL CONTACT - APPLICATIONS TO STEEL REINFORCED CONCRETE STRUCTURES
}

\author{
ROMAN VODIČKA* \& FILIP KŠIŇAN \\ Technical University of Košice, Faculty of Civil Engineering, Slovakia.
}

\begin{abstract}
A model for numerical analysis of compound structures made of various materials is presented. The mathematical concept of solution is based on quasi-static evolution of debonding processes occurring along the interface. It is formulated in terms of energies considering the stored energy represented by the elastic energy of the structures and dissipation due to damage processes, plastic slip at the interface or friction. The numerical solution includes a semi-implicit time stepping procedure, relying on splitting of the whole problem at a current time step into two problems of variational nature solved recursively. The space discretisation includes Symmetric Galerkin Boundary Element Method used to obtain the stored energies, and, in combination with the variational character of the recursive problems, also to calculate its gradients to be utilized in non-linear programming algorithms for finding the timeevolving solution. Numerical results are demonstrated for a steel-concrete interface frequently met in civil engineering applications to assess the model applicability in engineering practice.

Keywords: cohesive interface, contact with friction, interface damage, interface plastic slip, quadratic programming, quasi-static delamination, SGBEM
\end{abstract}

\section{INTRODUCTION}

Various engineering applications consider analysis of compound structures made of various materials. Numerical analysis of such structures which additionally includes, at least at the material interface, also damage mechanisms, plastic deformations or frictional contact is a challenging problem, which to be solved requires advanced computational techniques.

Computational simulations of damage evolution also with the other non-linear phenomena eventually lead to problems of crack propagation at such material interfaces. All of them are currently under intensive development. There exist several fracture models which, unlike classical fracture mechanics, enable also prediction of crack initiation: cohesive zone models (CZM) [1-3], finite fracture mechanics models [4-6], or variationally based interface damage and plasticity models [7-9].

Damage and subsequent cracking of an interface are usually modelled by an internal variable introduced on the interface. The concept of such damage variable was introduced by Frémond [10]. In the present model, interface stress-strain relations providing the structural response similar to known CZMs $[11,12]$ are considered and also other features that are typical for interface cracks are taken into account.

First, it is known that an interface crack propagating in the opening fracture mode usually dissipates much less energy than in the shearing mode $[13,14]$. Therefore, it is necessary to consider additional dissipated energy in shearing mode. This energy may come from developed plastic deformation in the vicinity of the evolving interface $[15,16]$, or the fracture energy may be dependent on the mode of arising crack throught the fracture mode-mixity angle $[8,17,18]$. Both of these possibilities are considered in the present model.

*ORCID: http://orcid.org/0000-0002-7702-3126 
Second, when modeling cohesive behaviour of an interface by CZMs, the normal contact conditions are often represented by additional non-damageable stiffness in compression. Such an approach assumes the contact surface exhibiting certain elastic response also under compression, e.g. due to its roughness, or due to the presence of a thin layer of an adhesive. This approach is also suitable for mathematical analysis, even if friction is present $[19,20]$.

Third, viscosity can be considered in the domains to expand applicability of the model to more general states than elastic deformation [21,22], and also to ensure uniqueness of the solution if a sufficiently dissipative model is used, e.g. Kelvin-Voigt, see [21], even if the elastic state is approximated by vanishing-viscosity solution concept [23].

The proposed mathematical approach is based on an energetic formulation looking for a kind of a weak solution, approximated by a time stepping procedure and by the Symmetric Galerkin Boundary Element Method (SGBEM) as in [11]. In the solution process, it utilizes nonlinear programming algorithms based on quadratic ones of [24].

In what follows, the model incorporating all mentioned phenomena is described briefly in Section 2, some aspects of the numerical solution concept are provided in Section 3. Finally, Section 4 shows some results obtained by the numerical solution. In this section some problems of civil engineering discussing the steel-concrete interfaces are presented: from the point of view of convergence of the used model in numerical solution and from practical aspect to asses the applicability of the model in practical simulations.

\section{DESCRIPTION OF THE MODEL}

We consider a bounded domain $\Omega$ split into several bodies by internal interfaces $\Gamma_{\mathrm{C}}$. For the sake of simplicity only two 2D bodies will be considered. These bodies are supposed to occupy subdomains $\Omega^{\eta} \subset \mathbb{R}^{2}(\eta=\mathrm{A}, \mathrm{B})$ with bounded Lipschitz boundaries $\Gamma^{\eta}$, Fig. 1 . However, it should be noted that the theory can be generalized to allow also 3D bodies. Let $\vec{n}$ denote the unit outward normal vector defined at smooth parts of $\Gamma$, let $\vec{s}$ denote the unit tangential vector such that it defines anti-clockwise orientation of $\Gamma$. A potential superscript index $\eta$ refers to the pertinent subdomain.

The contact zone $\Gamma_{\mathrm{C}}$ is defined as the common part of $\Gamma^{\mathrm{A}}$ and $\Gamma^{\mathrm{B}}$, i.e. $\Gamma_{\mathrm{C}}=\Gamma^{\mathrm{A}} \cap \Gamma^{\mathrm{B}}$. The Dirichlet and Neumann boundary conditions are defined on the outer boundary disjoint parts $\Gamma_{\mathrm{D}}$ and $\Gamma_{\mathrm{N}}$, respectively, at a current time $t$, cf. eqn (8) below. Additionally, $\Gamma_{\mathrm{D}}$ is considered far from the contact boundary, i.e. $\bar{\Gamma}_{\mathrm{D}} \cap \bar{\Gamma}_{\mathrm{C}}=\varnothing$.

The difference (the gap for displacements) on $\Gamma_{C}$ of the functions defined on $\Omega^{\mathrm{A}}$ and $\Omega^{\mathrm{B}}$ will be denoted by $\llbracket \cdot \rrbracket$. In particular, the gap of displacements on the contact boundary $\Gamma_{\mathrm{C}}$ means $\llbracket u \rrbracket:=\left.u^{\mathrm{A}}\right|_{\Gamma_{\mathrm{C}}}-\left.u^{\mathrm{B}}\right|_{\Gamma_{\mathrm{C}}}$. Further, we will use the gap of the normal and tangential displacements

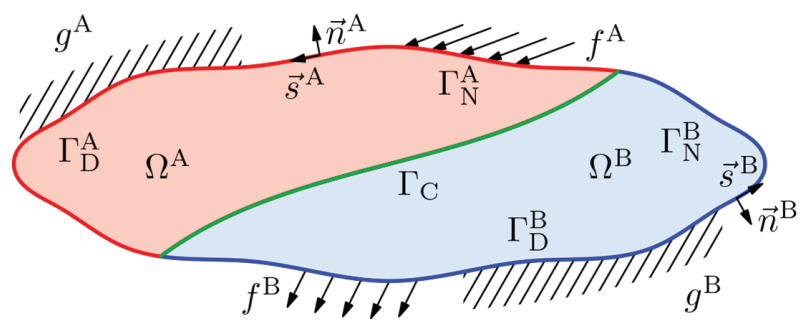

Figure 1: A sketch of two debonding domains. 


$$
\llbracket u \rrbracket_{\mathrm{n}}:=\llbracket u \rrbracket \cdot \vec{n}^{\mathrm{B}}=-\llbracket u \rrbracket \cdot \vec{n}^{\mathrm{A}}=-\left.u^{\mathrm{B}}\right|_{\Gamma_{\mathrm{C}}} \cdot \vec{n}^{\mathrm{B}}-\left.u^{\mathrm{A}}\right|_{\Gamma_{\mathrm{C}}} \cdot \vec{n}^{\mathrm{A}}, \quad \llbracket u \rrbracket_{\mathrm{s}}:=\llbracket u \rrbracket-\llbracket u \rrbracket \vec{n}^{\mathrm{B}} .
$$
We will also use the convention that te 'dot' will stand for the partial time derivative $\frac{\partial}{\partial t}$. The
model includes the force equilibrium on the contact interface $\Gamma_{\mathrm{C}}$ :

$$
\begin{gathered}
\llbracket \sigma \rrbracket \vec{n}=0 \quad \sigma:=\operatorname{De}(\dot{u})+\mathbb{C} e(u) \\
\sigma_{\mathrm{n}}-\kappa_{\mathrm{n}}(\zeta) \llbracket u \rrbracket_{\mathrm{n}}-\sigma_{\mathrm{c}}=0, \text { with } \sigma_{\mathrm{c}}:=-\kappa_{\mathrm{c}} \llbracket u \rrbracket_{\mathrm{n}}, \\
\left|\sigma_{\mathrm{f}}\right|<-\mu(\zeta) \sigma_{\mathrm{c}} \Rightarrow \llbracket \dot{u} \rrbracket_{\mathrm{s}}=0, \quad\left|\sigma_{\mathrm{f}}\right|=-\mu(\zeta) \sigma_{\mathrm{c}} \Rightarrow \exists \lambda \geq 0: \sigma_{\mathrm{f}}=\lambda \llbracket \dot{u} \rrbracket_{\mathrm{s}}, \\
\text { with } \sigma_{\mathrm{f}}:=\sigma_{\mathrm{s}}-\kappa_{\mathrm{s}}(\zeta)\left(\llbracket u \rrbracket_{\mathrm{s}}-\pi\right) \text {, and } \sigma_{\mathrm{s}}:=\sigma \vec{n}-\sigma_{\mathrm{n}} \vec{n} \text { with } \sigma_{\mathrm{n}}:=\vec{n}^{\mathrm{T}} \sigma \vec{n},
\end{gathered}
$$

where we consider a damageable interface represented by the normal and the tangential stiffnesses $\kappa_{\mathrm{n}}$ and $\kappa_{\mathrm{s}}$, respectively, depending on the scalar interface damage variable $\zeta$ limited to the range $[0 ; 1]$, with zero values pertinent to the total interface damage supposed to produce an interface crack. Various functions for this dependence were presented in [11] and [25], e.g. having $\kappa_{\mathrm{n}}(\zeta)=\kappa_{\mathrm{n} 0} \Phi(\zeta)$ and $\kappa_{\mathrm{s}}(\zeta)=\kappa_{\mathrm{s} 0} \Phi(\zeta)$ with

$$
\Phi(\zeta)=\frac{\beta \zeta}{1+\beta-\zeta}, \text { with } \beta>0
$$

leads to stress-strain relation of the bilinear CZM, [12].

There is also present the interface plastic slip $\pi$ on $\Gamma_{\mathrm{C}}$ and, simultaneously, we suppose frictional character of the contact between the subdomains with Coulomb friction law and damage dependent friction coefficient $\mu$ for which we prefer to use the relation.

$$
\mu(\zeta)=\mu_{0}(1-\zeta)^{p}, \text { with } \mu_{0}, p>0 .
$$

Furthermore, the flow rules for damage and plastic slip evolutions on $\Gamma_{\mathrm{C}}$ are considered:

$$
\begin{gathered}
\partial_{\zeta_{1}} a_{1}(\llbracket u \rrbracket, \dot{\zeta})+\frac{1}{2} \kappa_{s}^{\prime}(\zeta)\left|\llbracket u \rrbracket_{s}-\pi\right|^{2}+\frac{1}{2} \kappa_{n}^{\prime}\left(\zeta, \llbracket u \rrbracket_{n}\right)^{2}+N_{[0,1]}(\zeta) \ni \operatorname{div}_{\mathrm{s}}\left(\kappa_{2} \nabla_{\mathrm{s}} \zeta\right), \\
\dot{\pi} \in N_{\mathrm{l|| \leq \sigma _{y }}}\left(\operatorname{div}_{\mathrm{s}}\left(\kappa_{1} \nabla_{\mathrm{s}} \pi\right)-\kappa_{s}(\zeta)\left(\llbracket u \rrbracket_{\mathrm{s}}-\pi\right)+\kappa_{\mathrm{H}} \pi\right),
\end{gathered}
$$

With boundary conditions on $\partial \Gamma_{\mathrm{C}} \nabla_{\mathrm{s}} \pi \cdot \vec{n}_{\mathrm{s}}=0, \quad \nabla_{\mathrm{s}} \zeta \cdot \vec{n}_{\mathrm{s}}=0$,

where $N_{K}$ denotes the normal cone of the convex set $K$, see [8]. The $a_{1}$-term is supposed in the form

$$
a_{1}(\llbracket u \rrbracket ; \dot{\zeta}):=\left\{\begin{array}{cl}
-G_{\mathrm{c}}(\llbracket u \rrbracket) \dot{\zeta}, & \text { if } \dot{\zeta} \leq 0 \text { on } \Gamma_{\mathrm{C}}, \\
+\infty & \text { else, }
\end{array}\right.
$$

including the interface fracture energy $G_{\mathrm{c}}$, required to break a unit area of the interface, being considered to depend on the current state of the displacement jump $\llbracket u \rrbracket$. Any phenomenological law describing the dependence of $G_{\mathrm{c}}$ on current displacement gap can be used, say that of Benzeggagh [26], or Hutchinson [27]. 


$$
G_{\mathrm{c}}(\llbracket u \rrbracket)=G_{\mathrm{c}}^{\mathrm{I}}\left(1+\tan ^{2}\left(\frac{2}{\pi} \arctan \sqrt{\frac{G_{\mathrm{c}}^{\mathrm{II}}-G_{\mathrm{c}}^{\mathrm{I}}}{G_{\mathrm{c}}^{\mathrm{I}}} \arctan \mid \frac{\llbracket u \rrbracket_{\mathrm{s}}}{\llbracket u u \rrbracket_{\mathrm{n}}}}\right)\right) .
$$

The parameters $G_{\mathrm{c}}^{\mathrm{I}}$ and $G_{\mathrm{c}}^{\mathrm{II}}$ express the fracture energies in the pure Mode I (opening) and in the pure Mode II (shear), respectively.

The plastic slip in the interface, represented by the variable $\pi$, is initiated if the shearing stress reaches the the yield level $\sigma_{y}$ and continues evolving with kinematic hardening whose pertinent stiffness parameter is $\kappa_{\mathrm{H}}$.

In the domains, we consider the standard visco-elasticity (no inertia) using the Kelvin-Voigt rheology supplemented with standard boundary conditions:

$$
-\operatorname{div} \sigma=h \quad \text { in } \Omega \backslash \Gamma_{\mathrm{C}}, \quad u=g(t) \quad \text { on } \Gamma_{\mathrm{D}}, \quad \sigma \vec{n}=f(t) \quad \Gamma_{\mathrm{N}} .
$$

In addition, we consider an initial-value problem by prescribing the following initial conditions

$$
\left.u\right|_{t=0}=u_{0},\left.\quad \pi\right|_{t=0}=\pi_{0},\left.\quad \zeta\right|_{t=0}=\zeta_{0} .
$$

In the numerical solution, a variational structure of the described model is intended to be exploited. Therefore, let us briefly present energetics behind the boundary-value problem (BVP) eqns (2)-(9). The underlying overall free energy $\varepsilon=\varepsilon(u, \pi, \zeta)$ expressed here by mechanical internal energy can be split into its volumetric and interface parts as follows

$$
\varepsilon(u, \pi, \zeta):=\int_{\Omega \backslash \Gamma_{\mathrm{C}}} \frac{1}{2} \mathbb{C} e(u): e(u) \mathrm{d} x+\int_{\Gamma_{\mathrm{C}}} \psi(\llbracket u \rrbracket, \pi, \zeta) \mathrm{d} S,
$$

Where the specific interface energy $(\llbracket u \rrbracket, \pi, \zeta)$ is expressed as

$$
\psi(\llbracket u \rrbracket, \pi, \zeta):= \begin{cases}\frac{\kappa_{\mathrm{s}}(\zeta)}{2}\left(\llbracket u \rrbracket_{\mathrm{s}}-\pi\right)^{2}+\frac{\kappa_{\mathrm{n}}(\zeta)}{2}\left(\llbracket u \rrbracket_{\mathrm{n}}\right)^{2}+\frac{\kappa_{\mathrm{c}}}{2}\left(\llbracket u \rrbracket_{\mathrm{n}}^{-}\right)^{2} \\ +\frac{\kappa_{\mathrm{H}}}{2}|\pi|^{2}+\frac{\kappa_{1}}{2}\left|\nabla_{\mathrm{s}} \pi\right|^{2}+\frac{\kappa_{2}}{2}\left|\nabla_{\mathrm{s}} \zeta\right|^{2} & \text { if } 0 \leq \zeta \leq 1 \text { a.e. on } \Gamma_{\mathrm{C}}, \\ +\infty & \text { otherwise. }\end{cases}
$$

The other ingredient of the model is the overall pseudo-potential of dissipative forces $\mathscr{h}$, which also can be split into a volumetric and a surface contributions as follows:

$$
\begin{aligned}
\mathscr{R}(u, \zeta ; \dot{u}, \dot{\pi}, \dot{\zeta}):= & \int_{\Omega \backslash \Gamma_{\mathrm{C}}} \frac{1}{2} \mathrm{D} e(\overrightarrow{\dot{u}}): e(\dot{u}) \mathrm{d} x \\
& +\int_{\Gamma_{\mathrm{C}}} \mu(\zeta)\left|\kappa_{\mathrm{c}} \llbracket u \rrbracket_{\mathrm{n}}^{-}\right|\left|\llbracket \dot{u} \rrbracket_{\mathrm{s}}\right|+\sigma_{\mathrm{y}}|\dot{\pi}|+a_{1}(\llbracket u \rrbracket, \dot{\zeta}) \mathrm{d} S
\end{aligned}
$$

where the $a_{1}$-term, cf. eqn (6), represents the potential (and also the specific dissipation rate) of the rate-independent delamination process on the contact boundary $\Gamma_{\mathrm{C}}$.

Eventually, we need the (linear) functional of external mechanical loading $\mathscr{A}(\mathrm{t})$ which, after the standard transformation to time-constant (homogeneous) Dirichlet condition by the shift $u \rightarrow u+\bar{g}$ with $\bar{g}$ being an extension of $\mathrm{g}$ from eqn (8) on $\Omega$ is given by 


$$
\langle\mathscr{F}(t), u\rangle:=\int_{\Omega} h \cdot u-(\mathrm{D} e(\dot{\bar{g}})+\mathbb{C} e(\bar{g})): e(u) \mathrm{d} x+\int_{\Gamma_{\mathrm{N}}} f \cdot u \mathrm{~d} S .
$$

For simplicity and without eliminating interesting applications, we assume that $\Gamma_{\mathrm{D}}$ and $\Gamma_{\mathrm{C}}$ are far from each other so that one can assume $\left.\bar{g}\right|_{\Gamma_{\mathrm{C}}}=0$ and thus this shift transformation does not influence the flow rules on $\Gamma_{\mathrm{C}}$.

In terms of these functionals, the evolution BVP can be expressed by the system of nonlinear variational inclusions as:

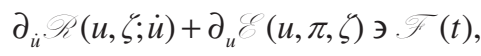

$$
\begin{aligned}
& \partial_{\dot{\pi}} \mathscr{R}(\dot{\pi})+\partial_{\pi} \mathscr{\ell}(u, \pi, \zeta) \ni 0 \\
& \partial_{\dot{\zeta}} \mathscr{h}(u ; \dot{\zeta})+\partial_{\zeta} \mathscr{C}(u, \pi, \zeta) \ni 0,
\end{aligned}
$$

where we reflected special form of $\mathscr{R}$ in relation to the used subdifferentials, e.g. $\partial_{\xi} \mathscr{R}$ does not depend on $\dot{u}$ and $\dot{\pi}$.

The system provides also energy balance relation. It can be obtained by testing eqns (13a), (13b), and (13c) by $\dot{u}, \dot{\pi}$, and $\dot{\zeta}$, respectively, which gives:

$$
\frac{\mathrm{d}}{\mathrm{d} t} \mathscr{C}(u, \pi, \zeta)+\mathscr{\gamma}(u, \zeta ; \dot{u}, \dot{\pi}, \dot{\zeta})=\langle F(t), \dot{u}\rangle
$$

Where $\mathscr{Y}(u, \zeta ; \dot{u}, \dot{\pi}, \dot{\zeta}):=\left\langle\partial_{\dot{u}} \mathscr{h}(u, \zeta ; \dot{u}), \dot{u}\right\rangle+\left\langle\partial_{\dot{\pi}} \mathscr{h}(\dot{\pi}), \dot{\pi}\right\rangle+\left\langle\partial_{\dot{\zeta} \mathscr{\ell}}(u ; \dot{\zeta}), \dot{\zeta}\right\rangle$ denotes the overall dissipation rate.

\section{DISCRETISATION}

Numerical solution requires both time discretization and spatial discretization. In what follows, we separately describe some aspects of both.

\subsection{A semi-implicit fractional-step time discretisation}

The time discretisation which ensures numerical convergence and stability of the obtained solution frequently includes decoupling of the system by a suitable fractional-step method. The splitting of the variables then should be done so that it guarantees separate convexity of the free energy $\mathscr{E}$ and additive splitting of the dissipation potential $\mathscr{R}$. Here, we apply splitting of the variables to $(u, \pi)$ and $\zeta$, assuming that $(\mathrm{u}, \pi) \rightarrow \zeta(\mathrm{u}, \pi, \zeta)$ is convex for all $\zeta$, and $\zeta \rightarrow \delta(\mathrm{u}, \pi, \zeta)$ is convex for all $(\mathrm{u}, \pi)$. This assumption leads to a two-step decoupled scheme, written in the form of eqn (13) as:

$$
\begin{gathered}
\tau \partial_{u_{\tau}^{k}} \mathscr{R}\left(u_{\tau}^{k-1}, \zeta_{\tau}^{k-1} ; \frac{u_{\tau}^{k}-u_{\tau}^{k-1}}{\tau}\right)+\partial_{u_{\tau}^{k}} \mathscr{C}\left(u_{\tau}^{k}, \pi_{\tau}^{k}, \zeta_{\tau}^{k-1}\right) \ni \mathscr{T}(k \tau), \\
\tau \partial_{\pi_{\tau}^{k}} \mathscr{R}\left(\frac{\pi_{\tau}^{k}-\pi_{\tau}^{k-1}}{\tau}\right)+\partial_{\pi_{\tau}^{k}} \mathscr{C}\left(u_{\tau}^{k}, \pi_{\tau}^{k}, \zeta_{\tau}^{k-1}\right) \ni 0, \\
\tau \partial_{\zeta_{\tau}^{k}} \mathscr{R}\left(u_{\tau}^{k}, \zeta_{\tau}^{k-1} ; \frac{\zeta_{\tau}^{k}-\zeta_{\tau}^{k-1}}{\tau}\right)+\partial_{\zeta_{\tau}^{k}} \mathscr{C}\left(u_{\tau}^{k}, \pi_{\tau}^{k}, \zeta_{\tau}^{k}\right) \ni 0,
\end{gathered}
$$


Where the rates have been approximated by the finite differences, e.g. $\dot{\zeta} \approx \frac{\zeta_{\tau}^{k}-\zeta_{\tau}^{k-1}}{\tau}$, where $\zeta_{\tau}^{k}$ denotes the solution at the instant $k \tau$ introduced by the time step $\tau$.

The decoupled scheme provides a variational structure to the solved problem, with two recursive minimisations in each time step. First, the minimisation of

$$
\mathscr{H}_{1}^{k}(u, \pi):=\tau \mathscr{h}\left(u_{\tau}^{k-1}, \zeta_{\tau}^{k-1} ; \frac{u-u_{\tau}^{k-1}}{\tau}, \frac{\pi-\pi_{\tau}^{k-1}}{\tau}\right)+\mathcal{C}\left(u, \pi, \zeta_{\tau}^{k-1}\right)-\left\langle\mathscr{F}\left(\tau^{k}\right), u\right\rangle
$$

provides $\left(u_{\tau}^{k}, \pi_{\tau}^{k}\right)$, and then the minimisation of

$$
\mathscr{H}_{2}^{k}(\zeta):=\tau \mathscr{R}\left(u_{\tau}^{k}, \zeta_{\tau}^{k-1} ; \frac{\zeta-\zeta_{\tau}^{k-1}}{\tau}\right)+\mathcal{\delta}\left(u_{\tau}^{k}, \pi_{\tau}^{k} \zeta\right)
$$

provides $\zeta_{\tau}^{k}$. The minimisation system eqn (16) is to be solved recursively for $k=1, \ldots, T / \tau$, starting from $k=1$ with

$$
u_{\tau}^{0}=u_{0}, \quad \pi_{\tau}^{0}=\pi_{0}, \quad \zeta_{\tau}^{0}=\zeta_{0} .
$$

It is important to see that the scheme eqn (15) has a variational character of both sub-problems for $\left(u_{\tau}^{k}, \pi_{\tau}^{k}\right)$ and for $\zeta_{\tau}^{k}$ with convex functionals which ensures existence of the numerical solution and which makes the numerical solution relatively simple.

In fact, the functional $\mathscr{E}$ is quadratic with respect to $(u ; \pi)$ so that various efficient numerical QP algorithms can be used in the solution, see [24] for a survey. Here, we have used Conjugate Gradient (CG) based algorithms with bound constraints [24] and implemented in a way similar to [16]. Considering $\delta$ with respect to $\zeta$, it is quadratic if the functions $\kappa(\zeta)$ in eqn (10b) are quadratic. Nevertheless, generally, e.g. also for the function in eqn (3), the function can be more general. In such a case, we apply the QP algorithm sequentially as in [11].

Additionally, energy balance from eqn (14) can be adopted for the used discretization when replacing the time derivatives by the time differences. Therefore, the tests of the pertinent inclusions in eqns (15a), (15b), and (15c) by $u_{\tau}^{k}-u_{\tau}^{k-1}, \pi_{\tau}^{k}-\pi_{\tau}^{k-1}$, and $\zeta_{\tau}^{k}-\zeta_{\tau}^{k-1}$, respectively, result in the following estimate:

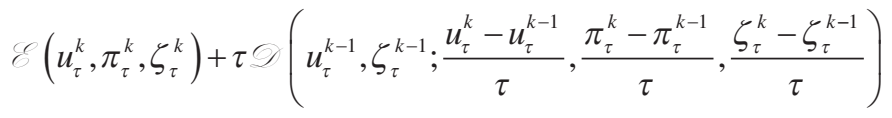

$$
\begin{aligned}
& \leq \mathcal{C}\left(u_{\tau}^{k-1}, \pi_{\tau}^{k-1}, \zeta_{\tau}^{k-1}\right)+\left\langle\mathscr{F}(k \tau), u_{\tau}^{k}-u_{\tau}^{k-1}\right\rangle
\end{aligned}
$$

which (im)balances the energies within the preformed time step and shows that the numerical solution does not have to conserve energy, even though the difference between the right-hand and the left-hand sides diminishes for refined discretisations. This relation can be verified by the numerical solution, cf. Fig. 3. 


\subsection{Notes on the spatial discretisation by SGBEM}

The viscosity as a part of the model is intentionally used especially for better mathematical treatment of the model, as it provides a convergence property of the discretized solution, even in the case of vanishing viscosity, though the convergence towards the inviscid solution can hardly be proved, see [23]. Additionally, it can be a natural part of the physical model, though the numerical approach requires a specific physical situation with a slight restriction by assuming that $\mathrm{D}^{n}=\tau_{r} \mathbb{C}^{n}$, where $\tau_{\mathrm{r}}>0$ is given relaxation-time parameter. We also suppose that the relaxation time $\tau_{\mathrm{r}}$ is the same for both subdomains and it is implemented by a simple trick from [22, 23]. It enables to reformulate the solution within the bulk domains in terms of an elastostatic problem solved by the conventional elastostatic SGBEM. The trick introduces a new fictitious displacement variable $v$ at the $k$-th time step by the relation

$$
v^{k}=u^{k}+\frac{\tau_{r}}{\tau}\left(u^{k}-u^{k-1}\right) .
$$

The functionals in eqn (16) are then reformulated in terms of the new variable $v$, see also [11].

The role of the SGBEM in the present computational procedure is to provide a complete boundary-value solution from the given boundary data to calculate the elastic strain energy in these domains. Thus, at each time step and at each iteration of the functional minimisation in eqn (16), the SGBEM code calculates unknown tractions $p$ along $\Gamma_{\mathrm{C}} \cup \Gamma_{\mathrm{D}}$ and unknown (fictitious) displacements $v$ along $\Gamma_{\mathrm{C}} \cup \Gamma_{\mathrm{N}}$, assuming the displacement gap $\llbracket v \rrbracket$ on $\Gamma_{\mathrm{C}}$ to be known from the used minimisation procedure.

The implementation of SGBEM, deduced from the energetic principles as shown in [28, 29], guarantees the positive-definite character of the computed strain energy. The present system of BIEs written in the weighted formulation can be arranged in the following block form, cf. [28, 29]:

$$
\left(\begin{array}{l}
\phi_{\mathrm{D}}^{\mathrm{A}} \\
\psi_{\mathrm{N}}^{\mathrm{A}} \\
\phi_{\mathrm{C}}^{\mathrm{A}} \\
\psi_{\mathrm{C}}^{\mathrm{A}} \\
\phi_{\mathrm{D}}^{\mathrm{B}} \\
\psi_{\mathrm{N}}^{\mathrm{B}} \\
\phi_{\mathrm{C}}^{\mathrm{B}} \\
\psi_{\mathrm{C}}^{\mathrm{B}}
\end{array}\right)^{\mathrm{T}}\left(\begin{array}{l}
\mathbf{K}^{\mathrm{A}} \\
\mathbf{M}^{\mathrm{AB}}
\end{array}\right.
$$$$
\mathbf{M}^{\mathrm{AB}}\left(\begin{array}{c}
p_{\mathrm{D}}^{\mathrm{A}} \\
v_{\mathrm{N}}^{\mathrm{A}} \\
p_{\mathrm{C}}^{\mathrm{A}} \\
v_{\mathrm{C}}^{\mathrm{A}} \\
p_{\mathrm{D}}^{\mathrm{B}} \\
v_{\mathrm{N}}^{\mathrm{B}} \\
p_{\mathrm{C}}^{\mathrm{B}} \\
v_{\mathrm{C}}^{\mathrm{B}}
\end{array}\right)=\left(\begin{array}{l}
\phi_{\mathrm{D}}^{\mathrm{A}} \\
\psi_{\mathrm{N}}^{\mathrm{A}} \\
\phi_{\mathrm{C}}^{\mathrm{A}} \\
\psi_{\mathrm{C}}^{\mathrm{A}} \\
\phi_{\mathrm{D}}^{\mathrm{B}} \\
\psi_{\mathrm{N}}^{\mathrm{B}} \\
\phi_{\mathrm{C}}^{\mathrm{B}} \\
\psi_{\mathrm{C}}^{\mathrm{B}}
\end{array}\right)^{\mathrm{T}}\left(\begin{array}{ll}
\mathbf{H}^{\mathrm{A}} & 0 \\
0 & \mathbf{H}^{\mathrm{B}} \\
& \\
&
\end{array}\right.
$$

With prescribed $\mathrm{w}=\llbracket v \rrbracket$ and with 


$$
\begin{aligned}
& \mathbf{K}^{\eta}=\left(\begin{array}{cccc}
-\mathbf{U}_{\mathrm{DD}}^{\eta} & \mathbf{T}_{\mathrm{DN}}^{\eta} & -\mathbf{U}_{\mathrm{DC}}^{\eta} & \mathbf{T}_{\mathrm{DC}}^{\eta} \\
\mathbf{T}_{\mathrm{ND}}^{\eta^{*}} & -\mathbf{S}_{\mathrm{NN}}^{\prime \prime} & \mathbf{T}_{\mathrm{NC}}^{\eta^{*}} & -\mathbf{S}_{\mathrm{NC}}^{\eta} \\
-\mathbf{U}_{\mathrm{CD}}^{\prime \prime} & \mathbf{T}_{\mathrm{CN}}^{\eta} & -\mathbf{U}_{\mathrm{CC}}^{\prime \prime} & \omega^{\prime \prime} \frac{1}{2} \mathbf{I}_{\mathrm{CC}}^{\eta}+\mathbf{T}_{\mathrm{CC}}^{\eta} \\
\mathbf{T}_{\mathrm{CD}}^{\eta^{*}} & -\mathbf{S}_{\mathrm{CN}}^{\prime \prime} & \omega^{\prime \prime} \frac{1}{2} \mathbf{I}_{\mathrm{CC}}^{\prime \prime}+\mathbf{T}_{\mathrm{CC}}^{\eta^{*}} & -\mathbf{S}_{\mathrm{CC}}^{\eta}
\end{array}\right), \quad \omega^{\eta}=\left\{\begin{array}{cc}
-1 & \text { if } \eta=A, \\
1 & \text { if } \eta=B,
\end{array}\right. \\
& \mathbf{H}^{\eta}=\left(\begin{array}{cc}
-\frac{1}{2} \mathbf{I}_{\mathrm{DD}}^{\eta}-\mathbf{T}_{\mathrm{DD}}^{\eta} & \mathbf{U}_{\mathrm{DN}}^{\eta} \\
\mathbf{S}_{\mathrm{DD}}^{\eta} & \frac{1}{2} \mathbf{I}_{\mathrm{NN}}^{\eta}-\mathbf{T}_{\mathrm{NN}}^{\eta^{*}} \\
-\mathbf{T}_{\mathrm{CD}}^{\eta} & \mathbf{U}_{\mathrm{CN}}^{\eta} \\
\mathbf{S}_{\mathrm{CD}}^{\eta} & -\mathbf{T}_{\mathrm{CN}}^{\eta^{*}}
\end{array}\right), \quad \mathbf{M}^{\mathrm{AB}}=\left(\begin{array}{cccc}
0 & 0 & 0 & 0 \\
0 & 0 & 0 & 0 \\
0 & 0 & 0 & \mathbf{I}_{\mathrm{CC}}^{\mathrm{AB}} \\
0 & 0 & 0 & 0
\end{array}\right) .
\end{aligned}
$$

The above equations use the (weakly singular) Kelvin fundamental solution $U_{i j}^{\eta}(x, y)$, and the associated derivatives obtained by the differential traction operator - the strongly singular function $T_{i j}^{\eta}(x, y)$ and the hypersingular function $S_{i j}^{\eta}(x, y)$. The compact form of BIEs in eqn (20) uses the notation

$$
\omega_{q}^{\eta \mathrm{T}} \mathbf{Z}_{q r}^{\eta} w_{r}^{\eta}=\int_{\Gamma_{q}^{\eta}} \omega_{j}^{w}(y)\left(\int_{\Gamma_{r}^{\eta}} Z_{j i}^{\eta}(y, x) w_{i}^{\eta}(x) \mathrm{d} S(x)\right) \mathrm{d} S(y),
$$

where $\omega$ stands for $\phi$ or $\psi$, while $w$ stands for $v$ or $p$, and further $q$ and $r$ stand for $\mathrm{D}, \mathrm{N}$, and $\mathrm{C}$, and eventually $Z^{\eta}$ stands for $U^{\eta}, T^{\eta}, T^{\eta *}$ or $S^{\eta}$, and where the inner integral can be regular, weakly singular, Cauchy principal value or Hadamard finite part integral. In the previous relations, $\mathbf{I}^{\eta}$ denotes the identity operator with the subscripts and superscripts specifying the part of the boundary where it is restricted to.

\section{NUMERICAL RESULTS}

The described model was used to analyse the compound structures made of steel and concrete. Two examples will be presented. In the first one, a standard four-point bending test is under consideration. Some numerical properties of the described model are presented, including convergence, influence of friction or of the bulk viscosity. The second example includes a T-shape connecting member to improve shearing connection between the two materials. In both examples, the elastic parameters are: $E_{s}=210 \mathrm{GPa}, v_{\mathrm{s}}=0.3$ for steel and $E_{c}=38 \mathrm{GPa}, \mathrm{v}_{\mathrm{c}}=0.2$ for concrete.

The geometrical model for the first example is shown in Fig. 2. The bottom layer is made from steel. The interface characteristics for the physical model based on eqns (3), (4), and (7) are $\kappa_{\mathrm{n} 0}=k_{\mathrm{s} 0}=10 \mathrm{TPam}^{-1}, \beta=0.2195, \kappa_{\mathrm{c}}=100 \mathrm{TPam}^{-1}, G_{\mathrm{c}}^{\mathrm{I}}=0.26 \mathrm{kJm}^{-2}, G_{\mathrm{c}}^{\mathrm{II}}=1.0 \mathrm{kJm}^{-2}$, if friction is considered then $\mu_{0}=0.8$ and $p=4$, and if viscosity is considered then $\tau_{\mathrm{r}}=0.01 \mathrm{~s}$ or $0.1 \mathrm{~s}$. Pleasetic slip is not taken into account, hence $\sigma_{\mathrm{y}}$ is large.

As we intend to demonstrate numerically convergence properties, various discretisations have been used. The basic time step is $\tau=0: 1 \mathrm{~s}$ which is refined by the factor 2 in subsequent discretisations. Accordingly, the space meshes are refined, where the smallest element is $10 \mathrm{~mm}$ long and the longest one has $50 \mathrm{~mm}$ in the coarsest boundary element mesh.

The loading is prescribed in displacements as shown in Fig. 2 by the simple linear function $g(t)=1 t \mathrm{mms}^{-1}$ up to maximal vertical shift $6 \mathrm{~mm}$. 


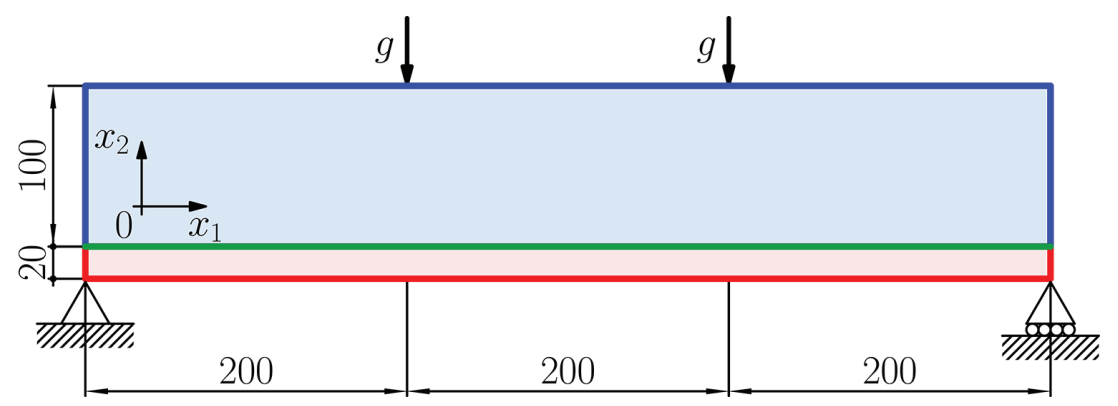

Figure 2: Geometry for the four-point bending example.

The results show how some global characteristics of the system are satisfied. First, satisfaction of the energy imbalance from eqn (18) is shown in Fig. 3. Naturally, refined discretisations, i.e. smaller $\tau$, make the difference to be smaller independently of friction or used viscosity time-relaxation parameter $\tau_{\mathrm{r}}$. Second, a working diagram for the beam showing the relation of the applied force on the prescribed displacement at the point of the right applied load is shown in Fig. 4. Generally, a tendency of the force distribution for more refined discretisations can be guessed. Also the influence of friction in the part (a) is evident, while the difference between viscid and inviscid calculation is not significant, cf. [23].

Finally, the results of the interface variables for the frictional case and given $\tau_{\mathrm{r}}$ are shown in Fig. 5 at the selected time instant. According to the graphs in Figs. 3(c) and 4(c), the extend of the crack represented by the domains where $\zeta=0$ is appropriate. The displacements are almost the same which means that the boundary elements converge satisfactorily.

The second example includes a T-shape connecting steel element shown in Fig. 6. Here, we consider interface characteristics different from those above. Friction is considered with
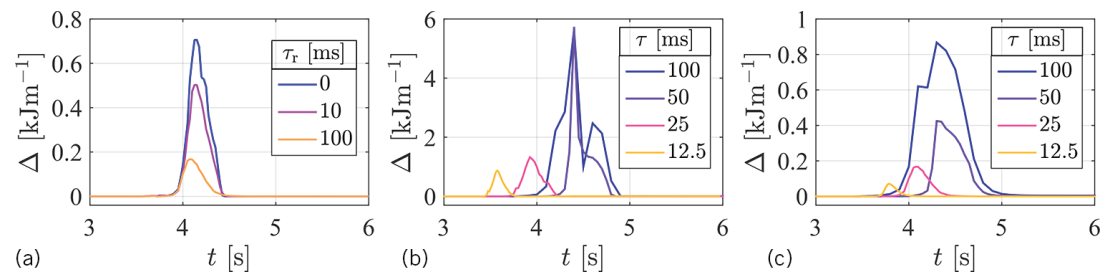

Figure 3: The energy imbalance of numerical solutions due to eqn (18): (a) various $\tau_{\mathrm{r}}, \tau=25$ ms, $\mu_{0}=0.8$; (b) $\tau_{\mathrm{r}}=0 \mathrm{~ms}$, various $\tau$, no friction; (c) $\tau_{\mathrm{r}}=100 \mathrm{~ms}$, various $\tau, \mu_{0}=0.8$.
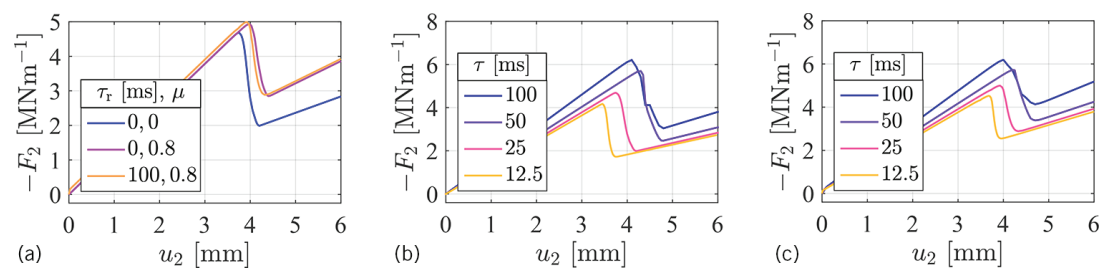

Figure 4: Working diagrams - the total vertical force $F_{2}$ applied at the point where acts one of the prescribed displacements $u_{2}=g$ : (a) various $\tau_{\mathrm{r}}$; (b) $\tau_{\mathrm{r}}=0 \mathrm{~ms}$, various $\tau$, no friction; (c) $\tau_{\mathrm{r}}=100 \mathrm{~ms}$, various $\tau, \mu_{0}=0.8$. 

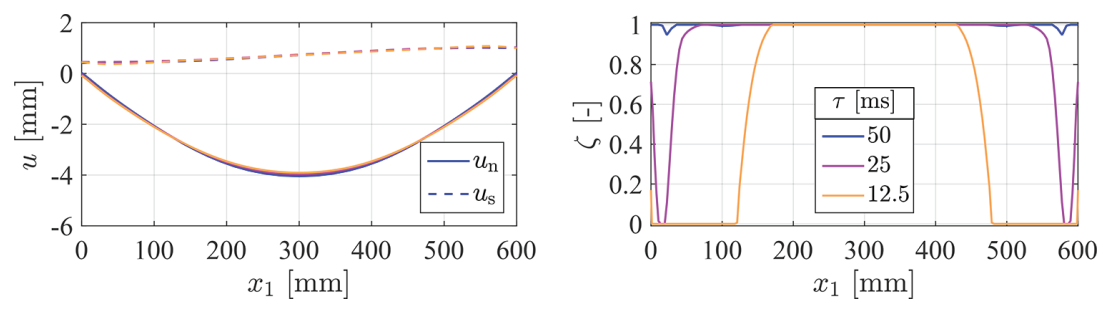

Figure 5: Distributions of interface variables for various discretisations (referred by $\tau$ ) at the instant $t=3.75 \mathrm{~s}, \tau_{\mathrm{r}}=100 \mathrm{~ms}, \mu_{0}=0.8$.
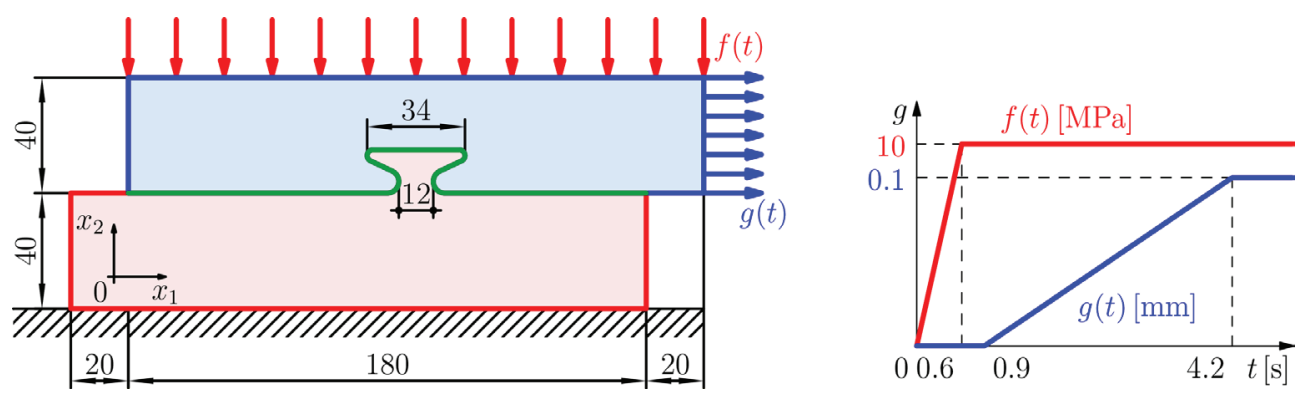

Figure 6: Geometry for the T-reinforcement example and the applied non-proportional loading.

$\mu_{0}=0.4$ and the same $p=4$ and viscosity is neglected, $\tau_{\mathrm{r}}=0 \mathrm{~s}$. The interface damage characteristics include constant fracture energy $G_{\mathrm{c}}=5 \mathrm{Jm}^{-2}$ and the interface plasticity is used with $\sigma_{\mathrm{y}}=1.25 \mathrm{MPa}$ and $k_{\mathrm{H}}=100 \mathrm{GPam}^{-1}$ to similate crack mode sensitivity.

The damage state of interface is described by the function, see [11]

$$
\Phi(\zeta)=\frac{\lambda_{1} \exp \left(-\gamma^{-1}(\alpha \zeta+\varepsilon)\right)-\lambda_{2}}{1-\lambda_{2}}, \quad \alpha=0.999, \varepsilon=0.0005
$$

with $\lambda_{1}=\exp \left(\gamma^{-1}(\alpha+\varepsilon)\right), \lambda_{2}=\lambda_{1} \exp \left(-\gamma^{-1}(\varepsilon)\right)$, and $\gamma(x)=\mathrm{e}^{-x}\left(1+x+\frac{1}{2} x^{2}\right)$ which, in terms of CZM, provokes stress-strain relation equivalent to the Ortiz-Pandolfi model [1]. The initial stiffness are $k_{\mathrm{n} 0}=k_{\mathrm{s} 0}=2 \mathrm{TPam}^{-1}, k_{\mathrm{c}}=200 \mathrm{TPam}^{-1}$.

The time step is set to $\tau=0: 02 \mathrm{~s}$. The boundary element mesh is non-uniform with refinements at the curved interface and at vicinity of subdomains' corner points, the smallest element is about $0.5 \mathrm{~mm}$ long.

The loading is considered non-proportional as it is shown in Fig. 6. First, the normal pressure is applied up to its maximal value of $10 \mathrm{MPa}$ and subsequently the horizontal displacements are applied at the top layer up to the maximal value of $0.1 \mathrm{~mm}$.

Some results are shown in Fig. 7. The displacements show the initial deformation after applying the normal pressure (a) and the final state of the structure (b). The evolution of the interface variables can be seen in the graphs below. The plastic slip starts to evolve first where the shearing stresses are important (around points $G$ and D). At the vicinity of the points B, $\mathrm{C}, \mathrm{E}$ and $\mathrm{F}$ the tangential stresses are small, so no plastic slip appears. 

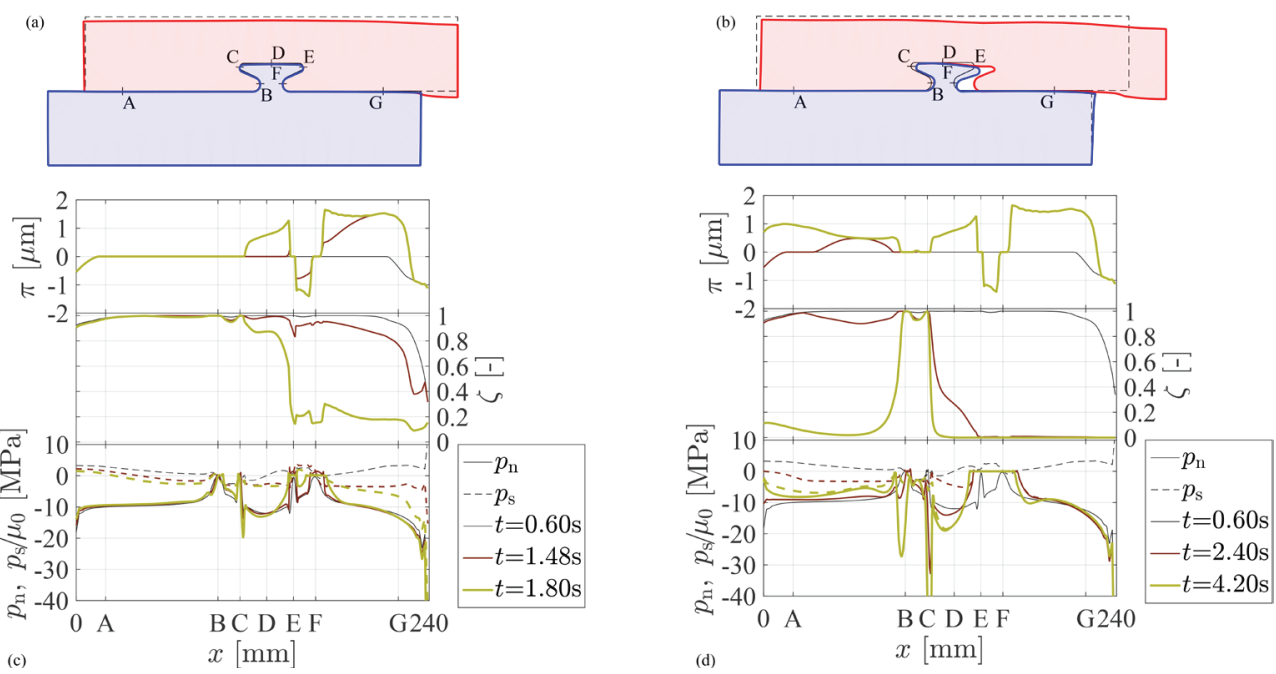

Figure 7: Results for the T-reinforcement example: (a) deformed shape at the instant $t=0.6 \mathrm{~s}$, (b) deformed shape at the instant $t=4.2 \mathrm{~s}$, (c) plastic slip, damage and interface stresses in the initial part of loading ( $\zeta$ positive), (d) plastic slip, damage and interface stresses in the final part of loading (interface crack evolving). The tangential stresses are scaled in order to see the zone where friction takes place. The displacements are magnified by a factor 200 .

The damage and subsequently the interface crack develops either due to shear around the point $\mathrm{G}$ or in the opening mode at the segment EF.

As the model incorporates also friction, the proposed friction function eqn (4) makes it significant only for substantially damaged interface ( $\zeta$ close to 0 ). When a shearing crack appears at the zone close to point $\mathrm{G}$, the influence of friction can be observed in the relation of the normal traction $p_{\mathrm{n}}$ and the tangential traction $p_{\mathrm{s}}$ where $\left|p_{\mathrm{n}}\right|=\left|p_{\mathrm{s}}\right| / \mu_{0}$.

Finally, the total force applied at the face where the displacement load is invoked is shown in Fig. 8. It documents the role of the T-shape reinforcement element as there is no weakening part in the force-displacement graph as we could see in Fig. 4.

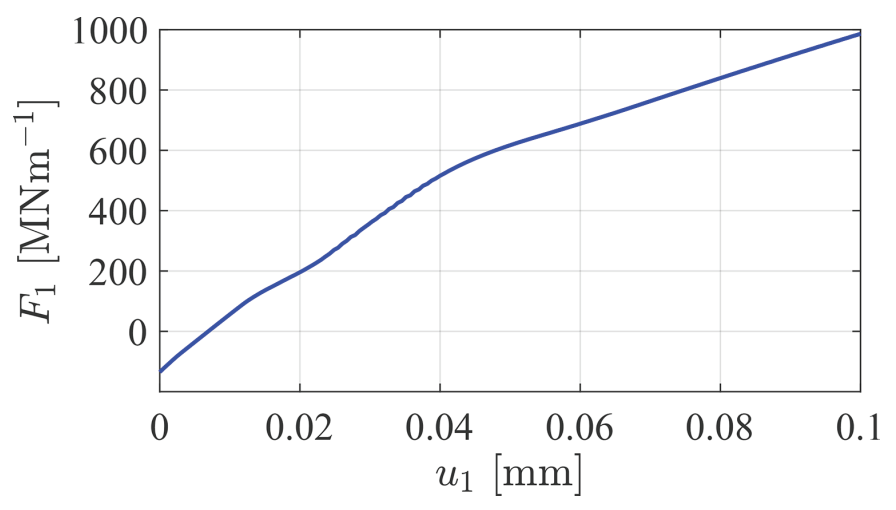

Figure 8: The force-displacement diagram of the total force applied at the right face of the upper body in the T-reinforcement example. 


\section{CONCLUSIONS}

An energy-based model for solving problems of interface cracks applied to some types of steel-concrete composite structures has been presented. The model includes such phenomena as interface damage, plastic slip and contact with friction. The results of numerical simulations show capabilities of the model to provide satisfactory results either from the mathematical point of view or from the point of view of application in engineering. The former was documented by some convergence properties observed in the first solved example, the latter is advocated by the analysis of a steel T-connector subject to pressure and shear in the other example. Obtained data confirm the influence of all observed phenomena and their importance in practical calculations. The presented numerical implementation confirmed the expected behaviour of the model in accordance with the applied theory. Its applicability for steel-concrete composite materials was satisfactorily demonstrated, too.

\section{ACKNOWLEDGEMENTS}

Authors acknowledge support from Slovak Research and Development Agency by the grant APVV-15-0486 and from the Slovak Ministry of Education by the grant VEGA 1/0078/16.

\section{REFERENCES}

[1] Ortiz, M. \& Pandolfi, A., Finite-deformation irreversible cohesive elements for three dimensional crack propagation analysis. International Journal for Numerical Methods in Engineering, 44, pp. 1267-1283, 1999. https://doi.org/10.1002/(sici)1097-0207(19990330)44:9<1267::aid-nme486>3.3.co;2-z

[2] Park, K. \& Paulino, G., Cohesive zone models: a critical review of traction-separation relationships across fracture surfaces. Applied Mechanics Reviews, 64(6), 2011. https://doi.org/10.1115/1.4023110

[3] Távara, L., Mantič, V., Salvadori, A., Gray, L. \& París, F., Cohesive-zone-model formulation and implementation using the symmetric Galerkin boundary element method for homogeneous solids. Computational Mechanics, 51, pp. 535-551, 2013. https://doi.org/10.1007/s00466-012-0808-5

[4] Leguillon, D., Strength or toughness? a criterion for crack onset at a notch. European Journal of Mechanics and Solids, 21(1), pp. 61-72, 2002. https://doi.org/10.1016/s0997-7538(01)01184-6

[5] Cornetti, P., Mantič, V. \& Carpinteri, A., Finite fracture mechanics at elastic interfaces. International Journal of Solids and Structures, 49, pp. 1022-1032, 2012. https://doi.org/10.1016/j.ijsolstr.2012.01.002

[6] García, I., Mantič, V., Blázquez, A. \& París, F., Transverse crack onset and growth in cross-ply $[0=90]_{\mathrm{S}}$ laminates under tension: application of a coupled stress and energy criterion. International Journal of Solids and Structures, 51, pp. 3844-3856, 2014.

[7] Kočvara, M., Mielke, A. \& Roubíček, T., A rate-independent approach to the delamination problem. Mathematics and Mechanics of Solids, 11, pp. 423-447, 2006. https://doi.org/10.1177/1081286505046482

[8] Roubíček, T., Kružík, M. \& Zeman, J., Delamination and adhesive contact models and their mathematical analysis and numerical treatment. Mathematical Methods and Models in Composites, ed. V. Mantič, Imperial College Press, pp. 349-400, 2013. https://doi.org/10.1142/9781848167858_0009 
[9] Raous, M., Cangemi, L. \& Cocu, M., A consistent model coupling adhesion, friction and unilateral contact. Computer Methods in Applied Mechanics and Engineering, 177(6), pp. 383-399, 1999. https://doi.org/10.1016/s0045-7825(98)00389-2

[10] Frémond, M., Dissipation dans l'adhérence des solides. CR Acad Sci, Paris, SérII, 300, pp. 709-714, 1985.

[11] Vodička, R., A quasi-static interface damage model with cohesive cracks: SQP-SGBEM implementation. Engineering Analysis with Boundary Elements, 62, pp. 123-140, 2016. https://doi.org/10.1016/j.enganabound.2015.09.010

[12] Vodička, R. \& Mantič, V., A multilinear cohesive law within a quasi-static interface damage model. Discrete and Continuous Dynamical Systems, 2017. (accepted).

[13] Banks-Sills, L. \& Askenazi, D., A note on fracture criteria for interface fracture. International Journal of Fracture, 103, pp. 177-188, 2000.

https://doi.org/10.1023/a:1007612613338

[14] Mantič, V., Discussion on the reference length and mode mixity for a bimaterial interface. Journal of Engineering Materials and Technology, 130, pp. 045501-1-2, 2008. https://doi.org/10.1115/1.2975232

[15] Panagiotopoulos, C., Mantič, V. \& Roubíček, T., BEM implementation of energetic solutions for quasistatic delamination problems. Computational Mechanics, 51, pp. 505-521, 2013. https://doi.org/10.1007/s00466-012-0826-3

[16] Vodička, R., Mantič, V. \& Roubíček, T., Energetic versus maximally-dissipative local solutions of a quasi-static rate-independent mixed-mode delamination model. Meccanica, 49(12), pp. 2933-296, 2014. https://doi.org/10.1007/s11012-014-0045-4

[17] Mantič, V. \& París, F., Relation between SIF and ERR based measures of fracture mode mixity in interface cracks. International Journal of Fracture, 130, pp. 557-569, 2004. https://doi.org/10.1023/b:frac.0000049496.77533.d5

[18] Roubíček, T., Panagiotopoulos, C. \& Mantič, V., Local-solution approach to quasistatic rate-independent mixed-mode delamination. Mathematical Models and Methods in Applied Sciences, 25(7), pp. 1337-1364, 2015. https://doi.org/10.1142/s0218202515500347

[19] Wriggers, P., Computational Contact Mechanics. Springer: Berlin, 2006.

[20] Vodička, R., Mantič, V. \& Roubíček, T., Quasistatic normal-compliance contact problem of visco-elastic bodies with Coulomb friction implemented by QP and SGBEM. Journal of Computational and Applied Mathematics, 315, pp. 249-272, 2017. https://doi.org/10.1016/j.cam.2016.10.010

[21] Roubíček, T., Panagiotopoulos, C. \& Mantič, V., Quasistatic adhesive contact of visco-elastic bodies and its numerical treatment for very small viscosity. Zeitschrift für Angewandte Mathematik und Mechanik, 93, pp. 823-840, 2013. https://doi.org/10.1002/zamm.201200239

[22] Panagiotopoulos, C., Mantič, V. \& Roubíček, T., A simple and efficient BEM implementation of quasistatic linear visco-elasticity. International Journal of Solids and Structures, 51, pp. 2261-2271, 2014. https://doi.org/10.1016/j.ijsolstr.2014.02.028 
[23] Roubíček, T., Adhesive contact of visco-elastic bodies and defect measures arising by vanishing viscosity. SIAM Journal on Mathematical Analysis, 45(1), pp. 101-126, 2013. https://doi.org/10.1137/12088286x

[24] Dostál, Z., Optimal Quadratic Programming Algorithms, volume 23 of Springer Optimization and Its Applications. Springer: Berlin, 2009.

[25] Vodička, R., Roubíček, T. \& Mantič, V., General-purpose model for various adhesive frictional contacts at small strains. Interf Free Bound, 2017. (submitted).

[26] Benzeggagh, M. \& Kenane, M., Measurement of mixed-mode delamination fracture toughness of unidirectional glass/epoxy composites with mixed-mode bending apparatus. Composites Science and Technology, 56, pp. 439-449, 1996. https://doi.org/10.1016/0266-3538(96)00005-x

[27] Hutchinson, J. \& Wu, T., Mixed mode cracking in layered materials. Advances in Applied Mechanics, 29, pp. 63-191, 1992. https://doi.org/10.1016/s0065-2156(08)70164-9

[28] Vodička, R., Mantič, V. \& París, F., Symmetric variational formulation of BIE for domain decomposition problems in elasticity - an SGBEM approach for nonconforming discretizations of curved interfaces. CMES - Computer Modeling in Engineering \& Sciences, 17(3), pp. 173-203, 2007.

[29] Vodička, R., Mantič, V. \& París, F., Two variational formulations for elastic domain decomposition problems solved by SGBEM enforcing coupling conditions in a weak form. Engineering Analysis with Boundary Elements, 35, pp. 148-155, 2011. https://doi.org/10.1016/j.enganabound.2010.05.002 